\title{
Analysis of ST-Segment Abnormalities in ECG using Signal Block Averaging Technique
}

\author{
S. Thulasi Prasad ${ }^{1}$, S. Varadarajan ${ }^{2}$ \\ Associate Professor, Dept. of ECE, CVSE, Tirupati, India ${ }^{1}$ \\ Professor, Dept. of ECE, SVUCE, Tirupati, India ${ }^{2}$
}

\begin{abstract}
The ischemic myocardium and coronary heart disease which occur due to abnormality in firing of the electrical impulses from the ventricles are causing an unanticipated death. This is most commonly referred to as Acute Myocardial Infarction (AMI). The value of ST-segment duration is not always easy to determine if the electrocardiograms (ECGs) are often spoiled by different types of artefacts. Occasionally it is not likely to recognize the exact end of the $\mathrm{S}$ wave and the accurate commencement of the $\mathrm{T}$ wave. Even if the Holter monitoring systems is useful to detect ST segment elevation or ST depression with good accuracy, there is no stringent technique of measuring an ischemic ST episode. This paper is intended to detect automatically ST-segment deviations and transient ST episodes which will help us in identification of Myocardial Infarction and also to categorize major cardiac arrhythmia. Our system has been tested and yielded an accuracy of $94.5 \%$ for the ST episode detection on the Long Term ST Database and 97.4\% on MIT-BIH Arrhythmia Database.
\end{abstract}

Keywords: ST segment, MI, FIR filter, QRS, QT interval

\section{INTRODUCTION}

The electrocardiogram (ECG - Electro Cardio Gram) [1] is a graphical record of bioelectrical signal generated by the human heart during the cardiac cycle and provides almost all information about the electrical activity of the heart. The ECG is used as a basic investigative and major diagnostic tool for the cardiologists in cardiology. It helps us to detect and analyse the Heart Rate Variability, Auricular and Ventricular Hypertrophy, Myocardial Infarction [2] (heart attack), Arrhythmias, Pericarditis and coronary artery disease in patients with chest pain syndromes. The information pertaining to these imperfections and defects are concentrated in intervals and magnitudes of the $\mathrm{P}$ wave, QRS complex, $\mathrm{T}$ wave, PR segment, QT interval, ST interval, PR segment, and ST segment of the ECG signal. Based on the above data, doctors can correctly diagnose human heart diseases. Therefore, analysing the ECG signals of cardiac Myocardial Infarction is very important for doctors to make correct clinical diagnoses and to take right decisions in right time.

The portion of the ECG that lies between the J-point end of the $S$ wave and beginning of the $T$ wave (Fig. 1) is known as ST segment [3]. This is not always flat instead it is elevated or depressed. Due to this variation it is not always easy to measure the duration of the ST segment. Sometimes it is not possible to identify the exact end of the $S$ wave and the exact beginning of the $T$ wave. Even if the ST deviation episodes (ST segment elevation or depression) [4] can be detected with good accuracy in Holter monitoring systems, there is no strict definition of an ischemic ST episode and it is quite challenging to unify very different approaches of detecting these episodes. In this paper a method was described to identify ST segment abnormalities more efficiently and to detect possible Myocardial Infarction.

\section{BACKGROUND}

A. Literature Review

The European Community in 1985 initiated an action to standardise the approach to detect and interpret ST segment and T-wave changes. In 1988, Aldrich et all developed an ECG score based on ST-segment deviation to quantify the size of the total risk in acute anterior and inferior myocardial infarction (AMI). The score was based on the assumption that the initial area of ischemia was an estimate of resultant infarct size in a population not receiving myocardial reperfusion therapy. In 1990 under the financial and scientific support of European Society of Cardiology ST-T database (ESC DB) (TADDEI et al., 1992b) was first released. The transient ST segment episodes of depression or elevation having amplitude greater than $100 \mathrm{mV}$ and the transient T-wave episodes of amplitude more than $200 \mathrm{mV}$ where documented as significant. Morabito and Silipo (MORABITO et al., 1992; SILIPO et al., 1993; 1995a) developed a number of recognition techniques to detect transient ischaemic events automatically in 1992 and 1993. A variety of neural network techniques (SILIPO et al., 1995b; SILIPO and MARCHESI, 1996; STAMKOPOULOS et al., 1998; MAGLAVERAS et al., 1998), a fuzzy-logic approach (PRESEDO et al., 1996b) and non-linear principal components analysis (DIAMANTARAS et al., 1996) were developed.

Between 1995 and 1996 Institute of Technology, in Cambridge, performed the analysis of ST-T alterations during myocardial ischaemia to address standardization. The investigation of the ESC DB had revealed an interesting temporal dynamics of transient ischaemic episodes (JAGER et al., 1996a), and a number of nonischaemic ST segment changes due to postural changes or slow drift of the ST deviation level (JAGER et al., 1995). Such non-ischaemic ST segment episodes may cause 
many false positive ischaemia detections when an automated analysis of transient ST events is performed.

An algorithm using Slope Vector Waveform (SVW) was designed to detect ECG QRS complex detection and RR interval evaluation by $\mathrm{Xu}$ et al. in [5]. In their proposed method the signal-to-noise ratio is improved by using variable stage differentiation for feature extraction, and the non-linear amplification. The method allows for a fast and accurate search of the R location, QRS complex duration, RR interval and yields excellent ECG feature extraction results. In order to get QRS durations, the feature extraction rules are needed.

Alexakis et al. in [6] illustrated a method to classify ECGs into normal and arrhythmic by automatically extracting both time interval and morphological features from the ECG. In this method a combination of Linear Discriminant Analysis (LDA) and artificial neural networks (ANN) techniques was used to extract features namely RR, RTc, and $\mathrm{T}$ wave amplitudes. The onset and end of the $\mathrm{T}$ wave were identified using the tangent method.

\section{B. Physiology}

The human heart is a cone-shaped muscular pump located in the mediastinal cavity of the thorax between the lungs and beneath the sternum. In a healthy heart a sequence of electrical impulses is generated by the natural pacemaker in the right atrium, which is called sinoatrial node (SA). This impulse sequence then flows down natural conduction pathways between the atria to the atrioventricular node and from there to both ventricles. Atria and ventricles contract in coordination as the impulses spread through the natural conduction paths in an orderly fashion. This process is accompanied by the transferring of blood from heart to the arteries and constitutes one cycle or period of the ECG waveform. This process can be viewed as follows: The sinus node, which is located near the entrance of the superior vena cava vein, acts as generator of the sinus rhythm. The sinus node starts the heartbeat cycle by depolarizing its cells and depolarizes nearby cells. This atrial depolarization is denoted as P-wave. The atria and the ventricles are electrically isolated from each other. Therefore, the activation potential has to travel through the atrioventricular node (AV-node), which acts like a bottleneck, decreasing propagation speed and resulting in PR interval of the ECG waveform. The time delay helps to fill the ventricles with blood. As the ventricles are getting filled up to the maximum extent, the impulse from the AV-node passes through bundle of His, bundle branches and then to Purkinje fibres. The electrical impulse then depolarizes the whole ventricle and causes contraction of the ventricles which squeezes blood into the arteries. This generates the QRS part of the ECG waveform. A slow repolarization of the ventricles after the contraction of the ventricles at the R-peak is represented by the $T$ wave.

A typical ECG tracing of the cardiac cycle (heartbeat) consists of a $\mathrm{P}$ wave, a QRS complex, a T wave, and a U wave, which is normally invisible in 50 to $75 \%$ of ECGs 


\begin{tabular}{|l|l|}
\hline QRS & $\begin{array}{l}\text { It represents the depolarization of the } \\
\text { ventricles and normally is around } \\
0.06 \text { to } 0.10 \text { sec (60 to 100 ms) in } \\
\text { duration. The QRS complex is larger } \\
\text { than the P wave because the } \\
\text { ventricles contain more muscle mass } \\
\text { than the atria. The QRS complex } \\
\text { looks "spiked" quite than rounded } \\
\text { due to the raise in conduction } \\
\text { velocity as the His and Purkinje } \\
\text { system coordinates the depolarization } \\
\text { of the ventricles. }\end{array}$ \\
\hline QT Interval & $\begin{array}{l}\text { It represents time extends from the } \\
\text { beginning of the QRS complex to the } \\
\text { end of the T wave and signifies the } \\
\text { ventricular depolarization and } \\
\text { repolarization. Varies according to } \\
\text { age, sex, and heart rate. }\end{array}$ \\
\hline ST - T wave & $\begin{array}{l}\text { It is a smooth, continuous waveform } \\
\text { beginning with the J-point (end of } \\
\text { QRS), and gradually rising to the } \\
\text { peak of the T and followed by a hasty } \\
\text { descent to the isoelectric baseline or } \\
\text { the onset of the U wave. }\end{array}$ \\
\hline T Wave & $\begin{array}{l}\text { It represents the repolarization (or } \\
\text { recovery) of the ventricles. The time } \\
\text { duration from the onset of the QRS } \\
\text { complex to the peak of the T wave is } \\
\text { termed as the absolute refractory } \\
\text { period and the last half of the T wave } \\
\text { is termed as the relative refractory } \\
\text { period (or vulnerable period) }\end{array}$ \\
\hline
\end{tabular}

\section{METHODOLOGY}

\section{A. Problem Formulation}

Standard tables are used as reference to relate the QT interval to the heart rate for measuring the control of ST segment changes on QT interval. If the ST segment is normal, the QT interval is usually normal. The QT interval [7] is normally prolonged due to bundle-branch block or pre-excitation of the ventricles. The QT interval is prolonged due to prolongation of the duration of the QRS complex and the ST segment. The QT interval prolongation is an indication to Hyperkalemia, Hypocalcemia and Hypothermia. So it is important to determine which components of QT interval are responsible for prolongation QT interval by performing statistical analysis on the ST segment/QT interval (ST/QT) slope [8].

\section{B. Problem Approach}

The raw ECG signal consists of original ECG and electrical noise, baseline drift and power line interference and needs to be filtered to uncover the ECG signal from behind this noise. The resolution of the signal can also be increased by averaging many waves over a length of time to form one ECG wave. Although standards for the filtering have been designated since 1975, an even higher resolution method is proposed in this paper by taking the average of multiple waveforms over a finite period of time than averaging the waveforms over the length of a signal. As a result, the different micro potentials can be taken into consideration without losing them as noise.

The baseline drift of raw ECG signal is normally rang between $0.05 \mathrm{~Hz}$ and $0.6 \mathrm{~Hz}$. First, we designed a linear phase filter FIR filter [9] with cut-off frequency of $0.5 \mathrm{~Hz}$ using Kaiser Window to remove this base line drift. The Fourier transform of the ECG signal has also been used to observe which frequencies are being removed. Secondly, we used a FIR filter to filler ECG signal with different cutoff frequencies between $90 \mathrm{~Hz}$ to $160 \mathrm{~Hz}$ to examine how the different parameters of the filtered signal will be affected [10]. We found that the cut-off frequency of 150 $\mathrm{Hz}$ is very suitable to smooth out the high frequencies without losing some of the physiological features and eliminate high frequency noise more effectively. The Fourier transform of the ECG signal has also been used to observe which frequencies are being removed by low pass filter. We used ST long-term Database as input ECG data signal and MATLAB 7.10 (R2010a) for testing and analysis. Lastly, the ECG signal is averaged using following signal averaging algorithm and computing ratio of ST/QT and AST/QT

\section{Algorithm \\ 1. Select $1 \mathrm{~min}$ ECG from long time ST database \\ 2. Split 1 min ECG into 10 blocks of each $6 \mathrm{sec}$ long \\ 3. Find QRS complex using Pan-Hopkins algorithm \\ 4. Select R-peak index of each block and find average of block \\ 5. Find $\mathrm{P}$ and $\mathrm{T}$ wave peaks using suitable window size from R-peak \\ 6. Find QT interval \\ 7. Find $\mathrm{J}$ and $\mathrm{K}$ points from each averaged block and find ST segment [11] \\ 8. Compute ST/QT and AST/QT for each block and plot}

\section{ST/QT ratio Method}

The steps 7 and 8 are important in our work. These can be performed as follows. In ECG wave he ST-T complex of signifies the time episode from the end of ventricular depolarisation to the end of the corresponding repolarisation in the electrical cardiac cycle. The ST segment forms a part of this wave complex starting with $\mathbf{J}$ point ending with the position of $\mathrm{K}$ point. The main aim of this algorithm is to prepare a procedure to detect $\mathrm{J}$ and $\mathrm{K}$ points and in turn perform ST/QT analysis [12]. We achieved our goal by determining the $\mathrm{J}$ point using the following formula.

$$
\mathrm{J}(\mathrm{n})=\mathrm{R}(\mathrm{n})+40+1.3 \sqrt{\mathrm{RR}(\mathrm{n}) \mathrm{ms}}
$$

Where $J(n)$ is beginning of ST segment corresponding to the $\mathrm{n}^{\text {th }}$ block, $\mathrm{R}(\mathrm{n})$ is the position of R-peak corresponding to the $\mathrm{n}^{\text {th }}$ block. The ST segment of each block is determined for 1 min ECG. 
Using ST segment and QT interval, the ST/QT ratio in $\mu \mathrm{V} / \mathrm{sec}$ is calculated. This ratio varies from block to block hence the maximum value of this ration is determined, which relates the deviation of ST segment elevation or depression in each block of ECG and the QT interval. The difference between ST/QT of each block and maximal $\mathrm{ST} / \mathrm{QT}$ in is calculated and represented as $\triangle \mathrm{ST} / \mathrm{QT}[13]$. Finally AST/MQT Index is calculated as the ratio of average of ST segments of all blocks to maximum QT. The Myocardial Infarction Sensitivity Index (MISI) is computed as the no of blocks whose ST/QT ratio exceeds AST/QT Index.

\section{Results}

The 50 subjects whose are having myocardial Infarction and age was 50 to 60 years were taken from MIT-BIH physionet database for our analysis. The ST/QT ratio is calculated for each $6 \mathrm{sec}$ block. The AST/QT ratio is computed to compare with each ST/QT ratio. The Myocardial Infarction sensitivity index (MISI) is determined for each subject. If MISI exceeds $70 \%$ of total no of blocks into which the ECG signal is split, the subject is declared as positive for Myocardial Infarction. If MISI is below $50 \%$ of total no of blocks into which the ECG signal is split, the subject is declared as negative for Myocardial Infarction. If the subject is not satisfying above both conditions then it is recommended for extensive trails over more no of blocks. The ECG record 's20041m' from MIT-BIH Long term ST Database (ltstdb) [14] for a period of 1 minute was taken and divided into 10 blocks each of $6 \mathrm{sec}$ duration. The first and $10^{\text {th }}$ block are shown plotted for in fig.2. The pre processed signal of one block after low pass and high pass filtering is also shown in fig. 2 .
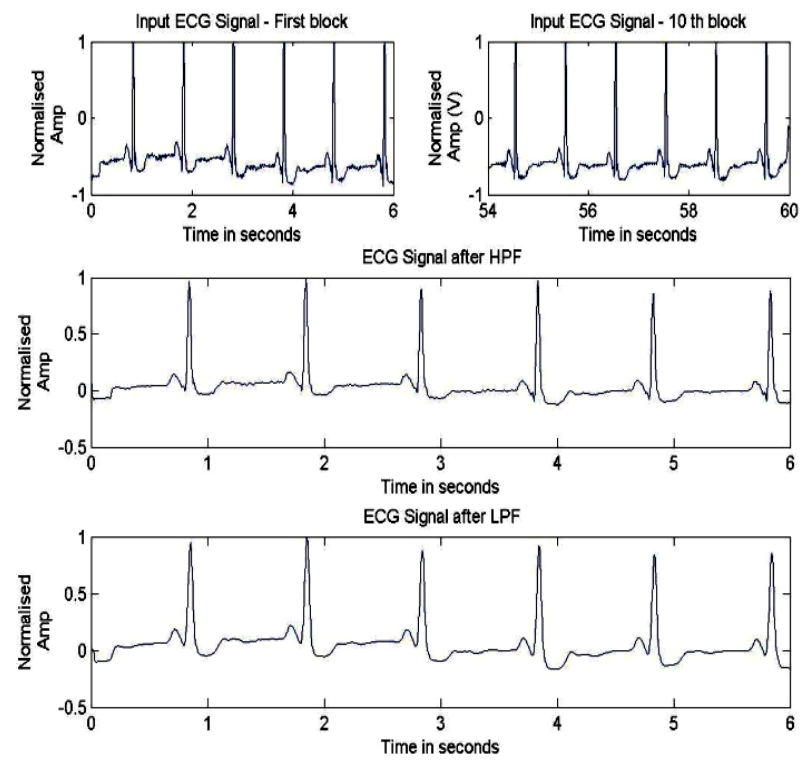

Fig. 2 First and Tenth block of ECG signal and pre-processed version of one block of ECG data

The fig.3 shows the QRS complex, $\mathrm{P}$ wave and $\mathrm{T}$ wave peaks of a block of ECG signal for a period of $6 \mathrm{sec}$.

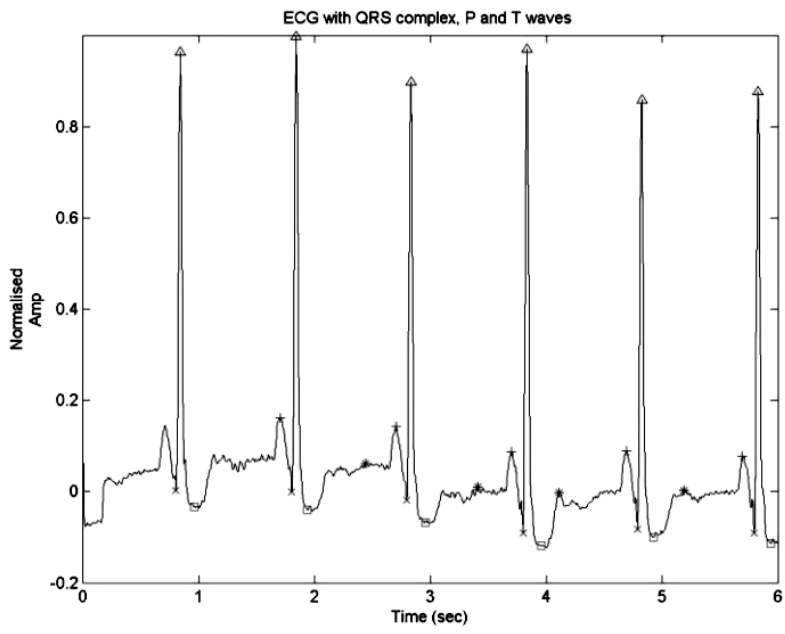

Fig. 3 QRS complex, P and T wave peaks of a block of ECG signal

The fig. 4 shows the average of 6 beats with $\mathrm{J}$ and $\mathrm{K}$ points. The magnitude frequency spectrum of ECG is also shown in the fig. 4.
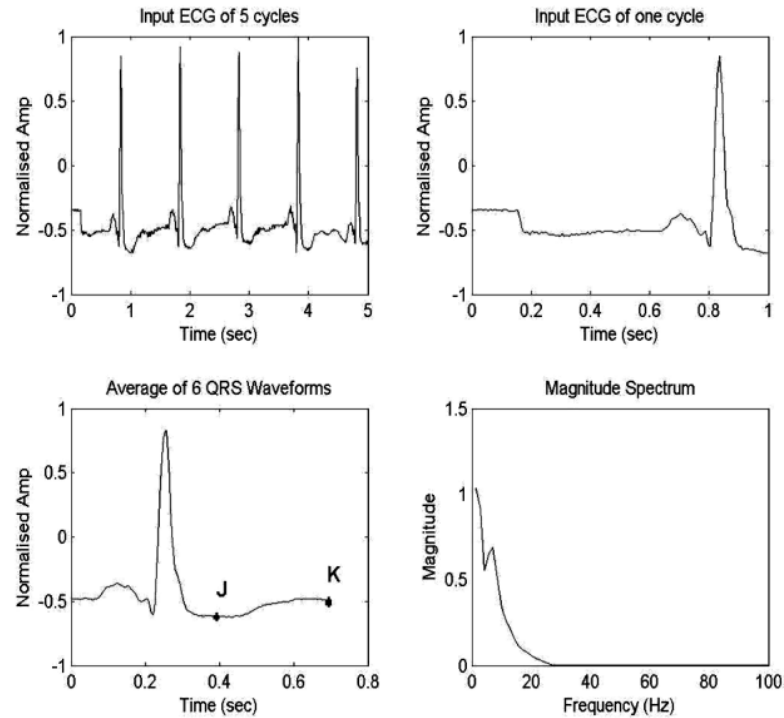

Fig. 4 A 5 beat and One beat ECG, Average of 6 beats and magnitude spectrum

\section{Conclusion}

The method presented here is very useful to decide whether a patient suffering from Myocardial Infarction or not if it is manifested by ST segment changes. The algorithm which we developed here in this paper can also be used for annotating ST change episodes which could be manually inspected. This method works well at low SNR of the ECG signal and gives better accuracy than any other method.

\section{ACKNOWLEDGMENT}

We gratefully acknowledge the Sree Vidyanikethan Engineering College, Tirupati for providing Laboratory facilities. We also extend our sincere thanks to SVRR hospital, Tirupati personal for providing basic clinical information regarding ECG. 


\section{REFERENCES}

[1] A. Camm, T. L. uscher, and P. Serruys, The ESC Textbook of cardiovascular Medicin,. OUP Oxford, 2009.

[2] Thygesen K, Alpert JS, White HD. Universal definition of myocardial infarction. Circulation 2007;116:2634.

[3] Ranjith P, Baby PC, Joseph P, "ECG analysis using wavelet transform: application to myocardial ischemia detection," ITBMRBM2003; 24: 44-47.

[4] Sivannarayana N, Reddy DC, "Biorthogonal wavelet transforms for ECG parameters estimation," Medical Engineering and Physics 1999; 21: 167-174.

[5] Xiaomin Xu, and Ying Liu, "ECG QRS Complex Detection Using Slope Vector Waveform (SVW) Algorithm," Proceedings of the 26th Annual International Conference of the IEEE EMBS, pp. 3597-3600, 2004. "PDCA12-70 data sheet," Opto Speed SA, Mezzovico, Switzerland.

[6] C. Alexakis, H. O. Nyongesa, R. Saatchi, N. D. Harris, C. Davies, C. Emery, R. H. Ireland, and S. R. Heller, "Feature Extraction and Classification of Electrocardiogram (ECG) Signals Related to Hypoglycaemia," Conference on computers in Cardiology, pp. 537-540, IEEE, 2003.

[7] Hayn, D., Kollmann, A., Schreier, G.: Automated QT interval measurement from multilead ECG signals. In: Computers in Cardiology, pp. 381-384. IEEE (2008)

[8] Lehtinen, R., "ST/HR Hysteresis: Exercise and Recovery Phase ST Depression/Heart Rate Analysis of the Exercise ECG," J. Electrocardiol., No. 32, 1999, pp. 198-204.

[9] Kaur, M., Singh, B., Ubhi, JS and Seema Rani. 2011. Digital Filteration of ECG Signals for Removal of Baseline Drift”, 2011 International Conference on Telecommunication Technology and Applications Proc .of CSIT vol.5 pg no.105-109.

[10] C. Vidal, V. Gatica, Design and Implementation of a Digital Electrocardiographic System, University of Antioquia Engineering Faculty Scientific Magazine, No. 55, (September 2010), pp. 99-107, ISSN 0120-0230, Antioquia, Colombia.

[11] Bacharova L, Mateasik A, Carnicky J, et al. The Dipolar ElectroCARdioTOpographic (DECARTO)-like method for graphic presentation of location and extent of area at risk estimated from ST-segment deviations in patients with acute myocardial infarction. J Electrocardiol 2009;42:172.

[12] Xiaozhong Chen and Natalia A. Trayanova, "A Novel Methodology for Assessing the Bounded-Input Bounded-Output Instability in QT Interval Dynamics: Application to Clinical ECG With Ventricular Tachycardia", IEEE Transactions On Biomedical Engineering, Vol. 59, No. 8, August 2012.

[13] A DSP Practical Application: Working on ECG Signal, Applications of Digital Signal Processing, Dr. Christian CuadradoLaborde (Ed.), ISBN: 978-953-307-406-1.

[14] PhysioNet, Physiobank, http://physionet.org/physiobank/database/

\section{BIOGRAPHIES}

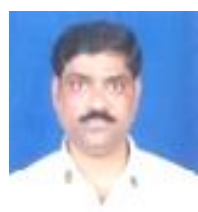

S. Thulasi Prasad received the M. Tech degree in Electronics and Communication Engineering from S. V. University College of Engineering, Tirupati, He has 16 years of teaching experience and 2 years of industrial experience. $\mathrm{He}$ is presently working in Sree Vidyanikethan Engineering College, Tirupati as Associate Professor.

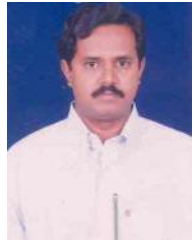

Dr. S. Varadarajan received the M. Tech degree in Electronics and Communication Engineering from NIT, Warangal and Ph.D degree from S. V. University College of Engineering, Tirupati. He has 23 years of teaching experience. He is presently working as Professor in the Dept. of ECE in S. V. University College of Engineering, Tirupati. 